\title{
Characterisation and cytotoxicity assessment of UV absorbers-intercalated zinc/aluminium-layered double hydroxides on dermal fibroblast cells
}

\begin{abstract}
$\mathrm{Zn}$ /Al-layered double hydroxide $(\mathrm{LDH})$ was used as a host to intercalate various organic ultraviolet (UV) radiation absorbers. The intercalation compounds were prepared via the coprecipitation method. Powder X-ray diffraction (PXRD) confirmed the successful intercalation of anions into the interlayer regions of the LDH nanocomposites. As a result of intercalation, the resulting nanocomposites loaded with UV-ray absorbers, cinnamic acid (CA), benzophenone 4 (B4) and Eusolex ${ }^{\circledR 232}$ (EUS) - exhibited basal spacings of $17.9 \AA$, 21.3 $\AA$ and 21.0 $\AA$, respectively. Photochemical analysis revealed an increase in the UV-ray absorption capability of UV absorber/LDH nanocomposites compared to pure UV-ray absorbers. The retention ability of the organic moieties in the LDH host was tested in a skin $\mathrm{pH}$ simulation and was found to demonstrate low release over an extended period of time. Cytotoxicity findings indicated that none of the nanocomposites exhibit significant cytotoxicity towards human dermal fibroblast (HDF) cells up to the test concentration of 25 $\mu \mathrm{g} / \mathrm{mL}$.
\end{abstract}

Keyword: Intercalation; Layered double hydroxide; Nanocomposite; Optical properties; UV-ray absorber 